\title{
In Search of Optimal Linkage Trees
}

\author{
Roy de Bokx \\ Delft University of Technology \\ Delft, The Netherlands \\ Rdebokx1990@gmail.com
}

\author{
Dirk Thierens \\ Utrecht University \\ Utrecht, The Netherlands \\ D.Thierens@uu.nl
}

\author{
Peter A.N. Bosman \\ Centrum Wiskunde \& \\ Informatica (CWI) \\ Amsterdam, The Netherlands \\ Peter.Bosman@cwi.nl
}

\section{Keywords}

Evolutionary Computation; Parallel Computation; Genetic Algorithms; Estimation-of-Distribution Algorithms; Linkage Learning; Optimal Mixing; Linkage Tree Genetic Algorithm

\section{CCS Concepts}

-Mathematics of computing $\rightarrow$ Evolutionary algorithms; •Computing methodologies $\rightarrow$ Model verification and validation;

\section{INTRODUCTION}

Linkage-learning Evolutionary Algorithms (EAs) use linkage learning to construct a linkage model, which is exploited to solve problems efficiently by taking into account important linkages, i.e. dependencies between problem variables, during variation. It has been shown that when this linkage model is aligned correctly with the structure of the problem, these EAs are capable of solving problems efficiently by performing variation based on this linkage model [2]. The Linkage Tree Genetic Algorithm (LTGA) uses a Linkage Tree (LT) as a linkage model to identify the problem's structure hierarchically, enabling it to solve various problems very efficiently. Understanding the reasons for LTGA's excellent performance is highly valuable as LTGA is also able to efficiently solve problems for which a tree-like linkage model seems inappropriate. This brings us to ask what in fact makes a linkage model ideal for LTGA to be used.

\section{OFFLINE LINKAGE TREE LEARNING}

To study the strengths and weaknesses of LTGA, we performed experiments aimed at learning LTs offline to be used as predetermined linkage models for LTGA, that replace the online-learned LTs $\left(\mathrm{LT}_{\text {on }} \mathrm{s}\right)$. Contrary to conventional approaches, this is done by searching in the space of LTs and evaluating the associated performance of LTGA.

Permission to make digital or hard copies of part or all of this work for personal or classroom use is granted without fee provided that copies are not made or distributed for profit or commercial advantage and that copies bear this notice and the full citation on the first page. Copyrights for third-party components of this work must be honored. For all other uses, contact the owner/author(s).

GECCO '15 July 11-15, 2015, Madrid, Spain

(C) 2015 Copyright held by the owner/author(s).

ACM ISBN 978-1-4503-3488-4/15/07.

DOI: http://dx.doi.org/10.1145/2739482.2764679
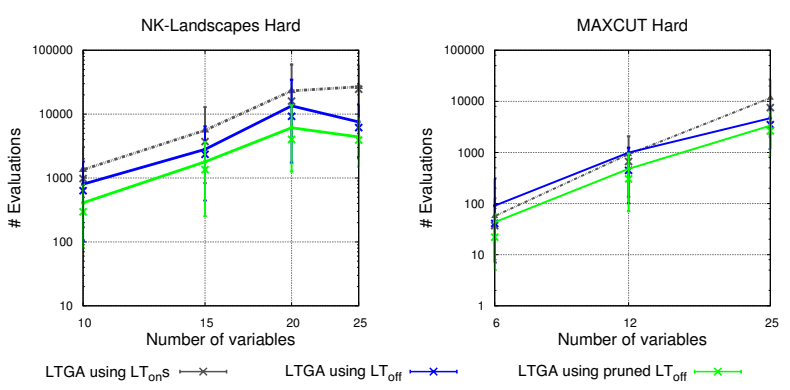

Figure 1: Experimental results of LTGA using (pruned) offline-learned LTs for the hardest $N K$ Landscapes and MAXCUT instances.

Using a binary encoding to represent all possible offlinelearned LTs $\left(\mathrm{LT}_{\text {off }} \mathrm{s}\right)$ is non-trivial and would result in a problem with high dimensionality, of which it is questionable whether it can be solved within reasonable time. Instead, the outcome of the hierarchical clustering algorithm used by LTGA to learn $\mathrm{LT}_{\text {on }} \mathrm{s}$ based on a population can be manipulated by forcing different contents into the distance matrix used, resulting in LTs that represent different linkage contexts. We used the real-valued EA known as iAMaLGaM to this end, as it was found to be very robust in finding suitable contents for a distance matrix [1]. The fitness of solutions, i.e. distance matrices, evaluated by iAMaLGaM was defined as the number of evaluations needed by LTGA with a newly implemented population-size-free scheme when the $\mathrm{LT}_{\text {on }} \mathrm{s}$ of LTGA are replaced by the corresponding offline-learned LT ( $\left.\mathrm{LT}_{\text {off }}\right)$, averaged over 1000 runs. This population-size-freescheme repeatedly initiates instances of LTGA, ever doubling the population size until a stopping condition is met.

This resulted in distance matrices that support the creation of optimal $\mathrm{LT}_{\text {off }} \mathrm{s}$, being LTs that cause the largest reduction in required number of evaluations by LTGA when such an $\mathrm{LT}_{\text {off }}$ is used as a predetermined linkage model, replacing the $\mathrm{LT}_{\text {on }} \mathrm{s}$ of LTGA.

\subsection{Experimental Results}

We compare the $\mathrm{LT}_{\text {off }} \mathrm{s}$ found by iAMaLGaM with the LT $T_{\text {on }} \mathrm{s}$ learned by the conventional LTGA in terms of LTGA's performance and by the contents of these LTs. Figure 1 shows the performance imposed by LTGA when using either $\mathrm{LT}_{\text {off }} \mathrm{s}$ or $\mathrm{LT}_{\text {on }} \mathrm{s}$, which corresponds to the fitness values found by iAMaLGaM. Results for pruned $L T_{\text {off }} s$ in Figure 1 can be ignored for now. 
For $N K$-Landscapes and MAXCUT, experiments show that LTGA using the $\mathrm{LT}_{\text {off }} \mathrm{s}$ substantially outperforms the conventional LTGA, as reflected by Figure 1. Moreover, LTGA using $\mathrm{LT}_{\text {off }} \mathrm{s}$ seems to be slightly better scalable, suggesting that the $\mathrm{LT}_{\text {off }} \mathrm{s}$ learned are intrinsically better than the $\mathrm{LT}_{\text {on }}$ and raising the question why this is the case.

Linkage sets in $\mathrm{LT}_{\text {off }} \mathrm{s}$ are determined based on LTGA's performance when using these $\mathrm{LT}_{\text {off }} \mathrm{S}$ and thus in a sense must contain key linkage structures. To obtain insight into the extent to which LTGA also identifies these structures, we study the overlap between the $\mathrm{LT}_{\text {off }} \mathrm{s}$ and the $\mathrm{LT}_{\text {on }} \mathrm{s}$ used by LTGA over 100 independent runs of LTGA. For Onemax, results of these experiments are rather trivial, however for Deceptive Trap, these experiments show that after the second generation all important linkage sets are present in the $\mathrm{LT}_{\text {on }}$ more than $98 \%$ of the time. This verifies that LTGA is able to identify important linkages and represent these in the LTs learned for problems with clear linkage structures.

While earlier attempts to construct appropriate predetermined linkage models were not fruitful for more intricate problems such as NK-Landscapes and MAXCUT [3], these results show that indeed such predetermined linkage models exist. This indicates that the LT on $\mathrm{s}$ used by LTGA might not be optimal linkage models as iAMaLGaM is able to learn $\mathrm{LT}_{\text {off }} \mathrm{s}$ that differ significantly from the $\mathrm{LT}_{\text {on }} \mathrm{s}$ while containing important linkage sets and supporting a better performance of LTGA. A clear explanation for this phenomenon was not found after inspecting the differences between $\mathrm{LT}_{\text {off }} \mathrm{s}$ and $\mathrm{LT}_{\text {on }} \mathrm{s}$.

\section{PRUNING THE LINKAGE TREE}

Experiments described above resulted in intrinsically better LTs. Though what if we take away the constraint of using a tree-structured linkage model? Additional overhead may exist in the LT in the form of superfluous linkage sets that impose additional evaluations when performing variation. Therefore, the found $\mathrm{LT}_{\text {off }} \mathrm{S}$ were pruned to filter out such linkage sets.

This was done with the use of LTGA itself by encoding subsets of an $\mathrm{LT}_{\text {off }}$ in a straightforward manner. For instance if the $\mathrm{LT}_{\text {off }}$ would be $\{\{0\},\{1\},\{2\},\{1,2\},\{0,1$, $2\}\}$, the solution 10010 would represent the linkage model $\{\{0\},\{1,2\}\}$. As no knowledge was available about the required population size for this problem, the population-sizefree scheme was used. Moreover, an internally parallelized implementation of LTGA was used in this scheme that is able to distribute the workload of the construction of the distance matrix and the generation of new solutions over processor cores available in a multi-core architecture.

\subsection{Experimental Results}

Experimental results show that LTGA, when using the selected subsets of the $\mathrm{LT}_{\text {off }} \mathrm{s}$ as linkage models, exhibits even better performance than LTGA when using the full $\mathrm{LT}_{\text {off }} \mathrm{s}$, further outperforming the conventional LTGA. The performance of LTGA when using the pruned $\mathrm{LT}_{\text {off }} \mathrm{S}$ is included in Figure 1 for the hardest problem instances of $N K$-Landscapes and $M A X C U T$ in a randomly generated test-suite of 100 instances.

An analysis of the contents of the pruned $\mathrm{LT}_{\text {off }} \mathrm{S}$ show that for Onemax and Deceptive Trap, the pruned LT ${ }_{\text {off }}$ contains all but one of the important linkage sets, which is as expected. For NK-Landscapes and MAXCUT, however, no clear correlation nor pattern could be found among the selected linkage sets. One clear pattern that was found in general, was that only half of the linkage sets in the $\mathrm{LT}_{\text {off }} \mathrm{s}$ were selected, which is likely due to the overlap between linkage sets contained in the LT. Experiments performed in which for each solution only a random half of the LT was used when performing variation, show that, although better than expected, the performance does not consistently exceed the performance of the conventional LTGA. This indicates that indeed a specific underlying scheme is causing half of the LTs to be redundant and a more advanced heuristic is needed to efficiently determine a more suitable linkage model for LTGA, either for fully learning the LT in a different manner of by pruning $\mathrm{LT}_{\text {on }} \mathrm{s}$ at low costs.

\section{CONCLUSIONS \& FUTURE WORK}

The recently introduced Linkage Tree Genetic Algorithm (LTGA) has been shown to exhibit excellent scalability on a variety of optimization problems. LTGA employs Linkage Trees (LTs) to identify and exploit linkage information between problem variables. Much is already understood about LTGA's performance, but it is still unclear whether the LT model can be further improved upon. In this work we analyzed the results of learning LTs offline by optimizing LTGA's performance as a function of static LTs. This resulted in a better performance of LTGA than with onlinelearned LTs as problem complexity increases. Further analysis of the offline-learned LTs indicated that pruning the LT can result in a further performance improvement of the LTGA up to a factor 6. Using a population-size-free internally parallelized version of LTGA, we found that the optimal subset of the offline-learned LT typically contains only about $50 \%$ of the nodes. This suggests that the LT model contains redundancies that may possibly still be exploited to improve the performance of LTGA with online-learned LTs. The magnitude of this performance improvement is subject to the costs implied by constructing improved linkage models, meaning that significant performance improvement can only be achieved when they can be constructed at costs that are comparable to the current costs of learning the LTs online. Future work is aimed at constructing a suitable metric for defining these exact costs of online linkage learning and gaining more insight into the contents of pruned $\mathrm{LT}_{\text {off }} \mathrm{s}$ in order to find a method for constructing linkage models of higher quality. This might ultimately enable us to reduce the number of evaluations, increasing the performance of LTGA and supporting the ability to solve more complex problems.

\section{REFERENCES}

[1] P. A. N. Bosman. On empirical memory design, faster selection of bayesian factorizations and parameter-free gaussian EDAs. In Proc. of the 11th Annual Conf. on Genetic and Evolutionary Computation, GECCO '09, pages 389-396, New York, NY, USA, 2009. ACM.

[2] P. A. N. Bosman and D. Thierens. More concise and robust linkage learning by filtering and combining linkage hierarchies. In Proc. of the 15th Annual Conf. on Genetic and Evolutionary Computation, GECCO '13, pages 359-366, New York, USA, 2013. ACM.

[3] D. Thierens and P. A. N. Bosman. Predetermined versus learned linkage models. In Proc. of the 14th Annual Conf. on Genetic and Evolutionary Computation, GECCO '12, pages 289-296, New York, USA, 2012. ACM. 